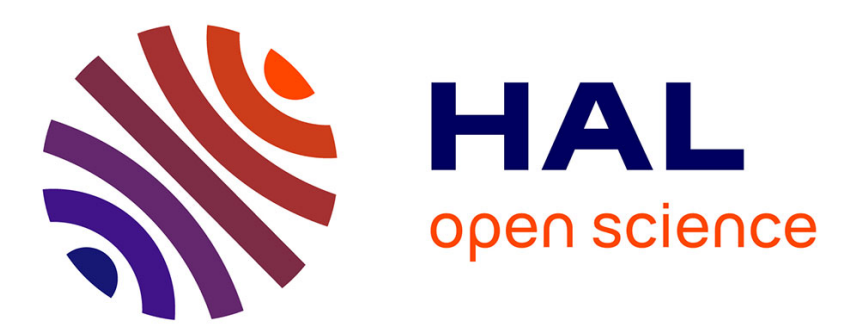

\title{
THE INFLUENCE OF CHROMATIC ABERRATION ON DEMOSAICKING
}

Xingbo Wang, Marius Pedersen, Jean-Baptiste Thomas

\section{To cite this version:}

Xingbo Wang, Marius Pedersen, Jean-Baptiste Thomas. THE INFLUENCE OF CHROMATIC ABERRATION ON DEMOSAICKING. 5th European Workshop on Visual Information Processing (EUVIP), 2014, Dec 2014, Paris, France. 10.1109/EUVIP.2014.7018410 . hal-01214039

\section{HAL Id: hal-01214039 \\ https://hal.science/hal-01214039}

Submitted on 9 Oct 2015

HAL is a multi-disciplinary open access archive for the deposit and dissemination of scientific research documents, whether they are published or not. The documents may come from teaching and research institutions in France or abroad, or from public or private research centers.
L'archive ouverte pluridisciplinaire HAL, est destinée au dépôt et à la diffusion de documents scientifiques de niveau recherche, publiés ou non, émanant des établissements d'enseignement et de recherche français ou étrangers, des laboratoires publics ou privés. 


\section{THE INFLUENCE OF CHROMATIC ABERRATION ON DEMOSAICKING}

\author{
Xingbo Wang ${ }^{* a, b}$, Marius Pedersen ${ }^{a}$ \\ ${ }^{a}$ Norwegian Colour and \\ Visual Computing Laboratory \\ Gjøvik University College, Gjøvik, Norway
}

\author{
Jean-Baptiste Thomas ${ }^{b}$ \\ ${ }^{\mathrm{b}}$ Laboratoire Electronique, \\ Informatique et Image \\ Université de Bourgogne, Dijon, France
}

\begin{abstract}
The wide deployment of colour imaging devices owes much to the use of colour filter array (CFA). A CFA produces a mosaic image, and normally a subsequent CFA demosaicking algorithm interpolates the mosaic image and estimates the full-resolution colour image. Among various types of optical aberrations from which a mosaic image may suffer, chromatic aberration (CA) influences the spatial and spectral correlation through the artefacts such as blur and mis-registration, which demosaicking also relies on. In this paper we propose a simulation framework aimed at an investigation of the influence of CA on demosaicking. Results show that CA benefits demosaicking to some extent, however CA lowers the quality of resulting images by any means.
\end{abstract}

Index Terms - demosaicking, chromatic aberration, colour filter array

\section{INTRODUCTION}

The advent of colour filter array (CFA) by Bayer [1] permits a single-sensor system capable of capturing a colour image at one exposure. The advantages mentioned above are gained at the expense of reduced spatial image resolution due to the intrinsic property of CFAs that merely one colour channel is sensed by each pixel. Therefore certain post processing becomes necessary to estimate other channels to a certain extent, known as demosaicking.

Optical images are commonly distorted by optical elements in form of various types of optical aberrations, and one of them resulting in colour artefacts is denoted chromatic aberration (CA) [2, p. 257]. It occurs because lenses, typically made of glass or plastic, bear different refractive indices for different wavelengths of light (the dispersion of the lens). The refractive index decreases with increasing wavelength. The main consequence of CA in imaging is that light rays at different wavelengths are focused at different image distances (axial/longitudinal CA) and at different locations in the image (transverse/lateral CA). In theory and reality only

\footnotetext{
*Send correspondence to xingbo.wang @ hig.no.
}

rays at a certain wavelength are focused accurately on the image plane, all other rays are focused before or behind the image plane, which leads to blur when observed from the image plane. Similarly, magnification of a lens varies from wavelength to wavelength as well. On the optical axis, there appears as axial CA, since magnification is zero. In a plane perpendicular to the optical axis, the difference of magnification rate turns more prominent as the distance from the optical axis increases, which produces transverse CA. The problem of CA may be corrected optically to some extent by using superior glass substrate whose refraction index varies to a lower degree and combining positive and negative lenses, nevertheless it is not avoidable in practice where cost, compactness, and weight matter.

CA influences the spatial and spectral correlations of images through the artefacts, such as blur and mis-registration, which also affect demosaicking. To the best of our knowledge, an evaluation of the influence of CA on demosaicking has not been performed. Therefore we address this issue in this work by building a modular framework that simulates the CFA imaging process.

The rest of the paper begins with a description of the experimental framework that includes an introduction to the simulation of CA in Section 2.1, followed by an explanation of the demosaicking algorithms under investigation in Section 2.2. Section 2.3 analyses the image quality metrics employed in the experiment, and the experimental setup is detailed in Section 2.4. Results are demonstrated and discussed in Section 3. Conclusions are drawn and future work is proposed in Section 4.

\section{EXPERIMENTAL FRAMEWORK}

The framework consists of four modules: CA simulation, CFA mosaicking, demosaicking and quality evaluation. Three of them are detailed in this section, while the conditions of CFA mosaicking are referred to in Section 2.4.

\subsection{Simulation of CA}

CA occurs in the formation of an optical image, therefore the best way of observing and studying $\mathrm{CA}$ is through various 
optical design. However, this is not flexible and convenient. In consequence, we opt to simulate CA with spectral images as an alternative solution. The two types of aberrations are simulated individually and jointly. The simulation of axial and transverse CAs are demonstrated in Fig. 1 and Fig. 3.

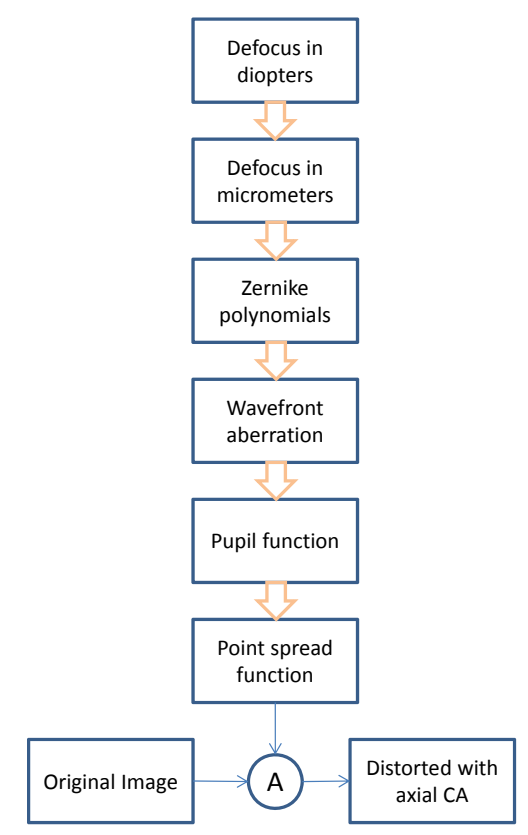

Fig. 1. Workflow for the simulation of axial CA.

Keeping in mind the reason for axial CA, we suppose the rays at $550 \mathrm{~nm}$ are focused on the sensor, such that green channel at $550 \mathrm{~nm}$ remains in focus and thus little changed, which is consistent with the peak in the CIE luminosity function. Then the rays at other wavelengths will be focused at a range of planes before and behind the intended image plane. This is seen as a blur. CA is an optical aberration closely bound to the optical design of a lens, and the degree of blur depends primarily on the position of the image plane for a given lens at given aperture size. It is often analysed in optical design and fabrication by means of ray tracing. However it varies from one optical design to another, and there is not a parametric model of such aberration to the best of our knowledge. To that end, we rely on the ISETBIO toolbox [3] that makes use of an ocular CA model $[4,5]$. With this reduced eye model, the chromatic refractive error, that is the dioptric difference of refraction, can be derived by Eq. 1,

$$
\Delta R_{E}(\lambda)=\frac{n\left(\lambda_{1}\right)-n\left(\lambda_{2}\right)}{r n_{D}}
$$

where $\lambda_{1}$ and $\lambda_{2}$ refer to the wavelengths in question, and $n\left(\lambda_{1}\right)$ and $n\left(\lambda_{2}\right)$, the refractive indices at these two wavelengths. $n_{D}$ is the refractive index for the sodium D-line (589 $\mathrm{nm}$ ), which is wavelength for which the model eye is emmetropic. $r$ is the corneal radius of curvature, set to $5.5 \mathrm{~mm}$. The refractive index is obtained by Eq. 2,

$$
n(\lambda)=a+\frac{b}{\lambda-c}
$$

where $a=1.320535, b=0.004685, c=0.214102$.

Now the defocus obtained is expressed in diopters. To make it suitable for the next step, which is the calculation of the pupil function, it is necessary to convert the defocus expressed in diopters to that expressed in micrometres by Eq. 3 .

$$
D_{m}=\frac{r^{2} D_{d}}{16 \sqrt{3}}
$$

where $r$ refers to the corneal radius of curvature in millimetres, and $D_{m}$ and $D_{d}$ denote defocus in micrometres and diopters respectively. The pupil function, or sometimes referred to as aperture function, is a complex function of the position in the pupil or aperture (an iris in this case) that represents the amplitude and phase of the wavefront across the pupil, also known as wavefront aberrations. It is an important tool to study optical imaging systems and their performance.

Optical system aberrations have historically been described, characterized, and catalogued by power series expansions, where the wave aberration is expressed as a weighted sum of power series terms that are functions of the pupil coordinates. Each term is associated with a particular aberration or mode, such as spherical aberration, coma, astigmatism, field curvature, distortion, and other higher order modes. Zernike polynomials form a complete set of functions or modes that are orthogonal over a circle of unit radius and are convenient for serving as a set of basis functions. Pupil functions are calculated here with the use of Zernike polynomials [6] and pupil function at a given wavelength is demonstrated in Eq. 4.

$$
P F(\lambda)=A e^{-i 2 \pi \frac{z}{\lambda}}
$$

where $A$ represents the amplitude, calculated entirely based on the assumed properties of the Stiles-Crawford effect, and the exponential function is actually the phase of the aberration. The Zernike polynomials, denoted by $\mathrm{Z}$, may consist of a series of modes, however, as the only aberration we are interested in this context is defocus ( $4^{\text {th }}$ mode), we simply neglect others.

The pupil function is related to the point spread function (PSF) by the Fourier transform [7, p. 131]. The reduced eye model simplifies the aberration and assumes the axial CA is shift-invariant, the generated PSF is static for each wavelength. An example of PSFs at a few wavelengths over the visible spectrum is demonstrated in Fig. 2. It is clear that the support of the PSFs vary in size as the wavelength increases, and achieves the minimum at $550 \mathrm{~nm}$. A PSF is in fact the impulse response of an imaging system in spatial domain, and the Fourier transform of a PSF leads to the optical transfer function that integrates the modulation transfer function (MTF) and the phase transfer function (PhTF). A convolution of a PSF and an image will produce the simulated image distorted by axial CA. 

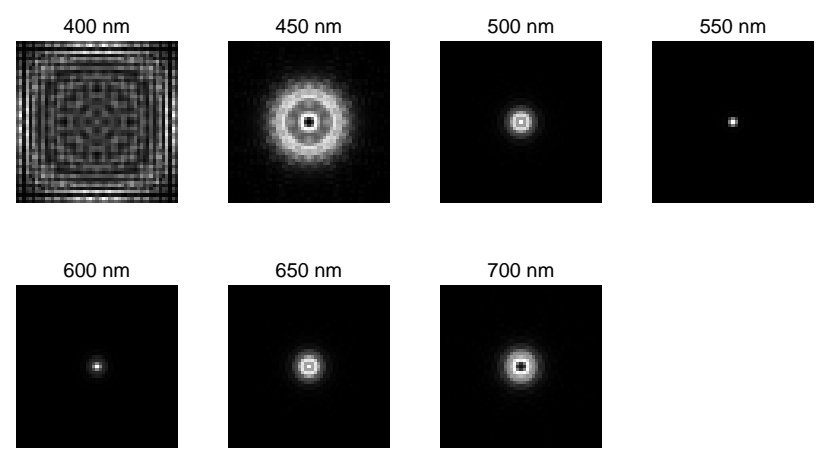

Fig. 2. A series of PSFs at visible wavelengths.

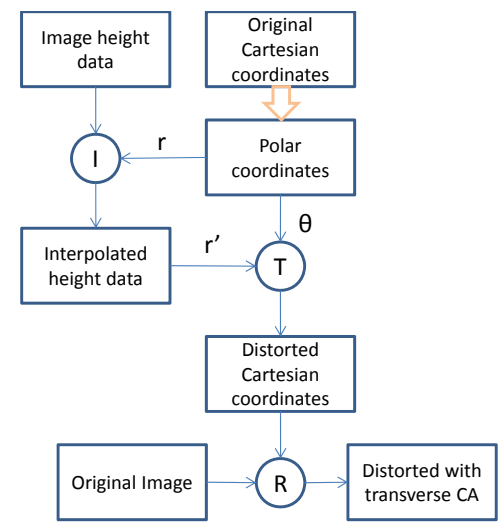

Fig. 3. Workflow for the simulation of transverse CA.

The mechanism of transverse CA lies in a varying rate of geometric distortion, that is in fact a radial operation depending on wavelengths. Therefore we first represent the images of each band in polar coordinates. Subsequently the radius can be manipulated in such a manner that the magnification varies in radial direction, following the ray tracing analysis of a real lens design. In practice, the measurement of transverse $\mathrm{CA}$ is often provided in form of a series of samples by the ray tracing software, which reflects the wavelength dependent radial distortion, as shown in Table 1. Accordingly, interpolation becomes essential to map and resample the original band images in order to simulate transverse CA.

As mentioned above, the reduced eye model simulates merely axial CA, and transverse CA depends on the results of ray tracing analysis. When combined, the two types of CA may be simulated sequentially as implemented by ISET [8]. Namely, transverse CA is applied prior to axial CA.

\subsection{Demosaicking algorithms under investigation}

Decades of research on CFA based imaging methods has resulted in a large number of evolving demosaicking algorithms. They can be well classified into two groups according to the intrinsic properties of the images they make use of. To be specific, some algorithms take advantage of spatial corre-

\begin{tabular}{|c|l|l|l|l|l|}
\hline \multirow{2}{*}{ Radial samples } & \multicolumn{5}{|c|}{ Distorted radial samples } \\
\cline { 2 - 6 } & $400 \mathrm{~nm}$ & $475 \mathrm{~nm}$ & $550 \mathrm{~nm}$ & $625 \mathrm{~nm}$ & $700 \mathrm{~nm}$ \\
\hline 0.0518 & 0.0507 & 0.0510 & 0.0511 & 0.0512 & 0.0513 \\
\hline 0.1037 & 0.1014 & 0.1019 & 0.1022 & 0.1024 & 0.1025 \\
\hline 0.1555 & 0.1519 & 0.1527 & 0.1531 & 0.1534 & 0.1537 \\
\hline 0.2073 & 0.2023 & 0.2033 & 0.2039 & 0.2044 & 0.2047 \\
\hline 0.2591 & 0.2525 & 0.2538 & 0.2545 & 0.2551 & 0.2554 \\
\hline 0.3110 & 0.3025 & 0.3040 & 0.3049 & 0.3055 & 0.3060 \\
\hline 0.3628 & 0.3521 & 0.3539 & 0.3549 & 0.3557 & 0.3562 \\
\hline 0.4146 & 0.4014 & 0.4034 & 0.4046 & 0.4054 & 0.4061 \\
\hline 0.4664 & 0.4503 & 0.4525 & 0.4539 & 0.4548 & 0.4555 \\
\hline 0.5183 & 0.4987 & 0.5012 & 0.5028 & 0.5038 & 0.5046 \\
\hline 0.5701 & 0.5467 & 0.5495 & 0.5511 & 0.5523 & 0.5531 \\
\hline 0.6219 & 0.5942 & 0.5972 & 0.5990 & 0.6003 & 0.6012 \\
\hline 0.6737 & 0.6411 & 0.6443 & 0.6463 & 0.6477 & 0.6487 \\
\hline 0.7256 & 0.6874 & 0.6909 & 0.6930 & 0.6945 & 0.6956 \\
\hline 0.7774 & 0.7330 & 0.7368 & 0.7391 & 0.7407 & 0.7418 \\
\hline 0.8292 & 0.7780 & 0.7821 & 0.7846 & 0.7862 & 0.7875 \\
\hline 0.8810 & 0.8224 & 0.8267 & 0.8293 & 0.8311 & 0.8324 \\
\hline 0.9329 & 0.8660 & 0.8705 & 0.8734 & 0.8753 & 0.8767 \\
\hline 0.9847 & 0.9089 & 0.9137 & 0.9167 & 0.9187 & 0.9202 \\
\hline 1.0365 & 0.9511 & 0.9561 & 0.9593 & 0.9614 & 0.9629 \\
\hline
\end{tabular}

Table 1. Positions of radial samples before and after transverse CA.

lation merely within one channel, whereas others benefit from spectral correlation between colour channels. Here we implemented and experimented with two representative methods, namely bilinear interpolation and gradient-corrected linear interpolation. The former relies on merely spatial correlation, whereas the latter also makes use of, and thus sensitive to, spectral correlation.

Indicated by its name, bilinear interpolation is an extension of linear interpolation for interpolating functions of two variables (e.g., $\mathrm{x}$ and y) on a regular $2 \mathrm{D}$ grid. In fact, bilinear interpolation estimates the unknown values by means of a distance weighted average of its neighbouring pixels. It is applied to each spectral band individually, as a result, it should be sensitive to variation of spatial correlation, however insensitive to alteration of spectral correlation.

Gradient based demosaicking solutions are aimed at reduced artefacts by avoiding interpolating across the edges. Malvar et al. [9] advance a gradient-corrected bilinear interpolated approach, with a gain parameter to control how much correction is applied. In other words, the results of bilinear interpolation is corrected by a measure of the gradient for the known colour at the pixel location. To determine appropriate values for the gain parameters, a Wiener approach is employed which computes the values leading to minimum meansquare errors, given second order statistics computed from the Kodak data set [10].

\subsection{Evaluation of demosaicking performance}

Evaluation of the demosaicking algorithms can be seen in general as an image quality evaluation task.

In the last decade when demosaicking gains much attention, the evaluation of demosaicking also attracts the interest of the academic community. Longère et al. conduct perceptual quality evaluation on a few demosaicking approaches, 
and state that perceptual results cannot be easily predicted using an image metric [11]. Even so, there are several attempts for objective quality evaluation. Lu and Tan propose two types of quality measures specifically for demosaicking, one computes the PSNR (peak signal-to-noise ratio) and CIE $\Delta E_{a b}^{*}$ for edge and smooth regions respectively, whereas the other one deals in particular with the zipper artefact [12]. Yang et al. show that low-level features (colours or edges) extracted from demosaicked images are affected by resolution, whatever the demosaicing method used, and therefore propose new criteria designed for low-level image analysis [13].

The most widely used criteria for the evaluation of demosaicing quality are MSE (mean squared error) and PSNR, primarily because they are simple thus easy to implement. However, the PSNR criterion provides a general estimation of the demosaicking quality, but does not really reflect the human judgement. Alternatives such as CIE $\Delta E_{a b}^{*}$ and SCIELAB [14] are also widely used, however they require apriori information like the reference white or illuminant [15].

In this work, we concern mostly the signal fidelity of each colour channel. Therefore we base our judgement on PSNR. In comparison with PSNR, the Structural SIMilarity (SSIM) [16] index provides more structural information and tends to be more consistent with subjective image quality assessment, which matches CA that blurs and shifts structures. Therefore we also adopted the SSIM as a method for measuring the similarity between the images before and after demosaicking.

In contrast to straightforward assessment of demosaicking methods or image quality, an evaluation of the influence of CA on demosaicking seems a bit more complicated, as there are two types of source images, i.e., with and without CAs. In consequence, with the two full reference quality metrics, we not only compute the image quality between the source and demosaicked images with and without distortions, but also cross compare the non-distorted source images and distorted demosaicked images.

\subsection{Experimental setup}

The experiment was conducted in the following manner. First, simulated CA was applied to spectral images separately. Then, a mosaic image is formed by filtering the distorted spectral image with a certain CFA mosaic and converting it to sRGB colour space. Next, the mosaic image was interpolated with one of the demosaicking methods. Finally, the demosaicked images were fed into a quality metric channel by channel.

Conditions and parameters in relation to the experiment are presented as follows. and the images were cropped into square matrices by the shorter dimension. Spectral range was set to $400 \mathrm{~nm}$ to $700 \mathrm{~nm}$, with an interval of $10 \mathrm{~nm}$. The CFA conforms to the common design of Bayer. Transverse CA was simulated according to real lens data provided by the ISET [8]. The SSIM index was computed with the empirical
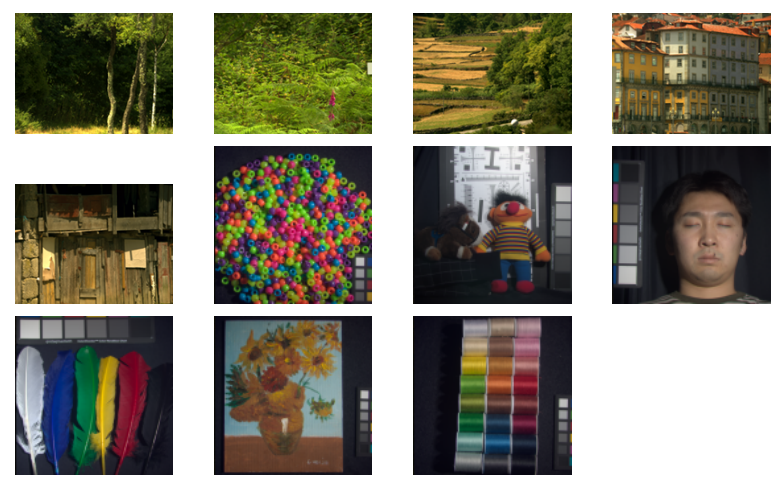

$$
\begin{array}{c|c|c|c}
1 & 2 & 3 & 4 \\
\hline 5 & 6 & 7 & 8 \\
\hline 9 & 10 & 11 &
\end{array}
$$

Fig. 4. Thumbnails of the images used. Scene 1-5 were selected from the Foster set [17] and scene 6-11 from the CAVE set [18].

formula specified on the official webpage to determine the scale for images viewed from a typical distance [19].

\section{RESULTS AND DISCUSSION}

Experimental results are depicted below. Lines and markers in red, green and blue represent the results for the corresponding channels, and those in black indicate the average values. Due to the space limitation, some similar results are not shown here. Concerning the performance with and without CAs, Fig. 5-6 show the results in terms of PSNR, while Fig. 7-8 display the results in terms of SSIM index. Similarly, Fig. 9-10 demonstrate the results in terms of PSNR and SSIM respectively for the cross comparison between non-distorted sources and distorted demosaicked images.

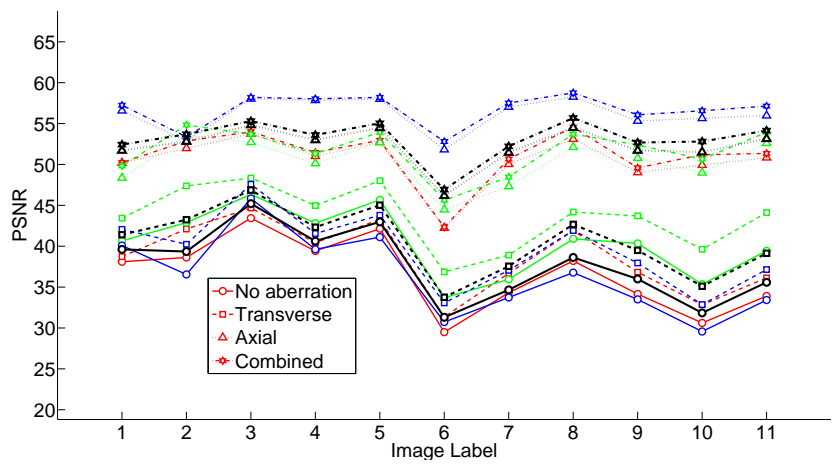

Fig. 5. PSNR computed from straight comparisons between distorted source images and distorted demosaicked images for $\mathrm{R} / \mathrm{G} / \mathrm{B}$ channels and the average with bilinear interpolation.

It can be seen from Fig. 5-6 that combined and axial CA yield higher performance, regardless of the demosaicking 


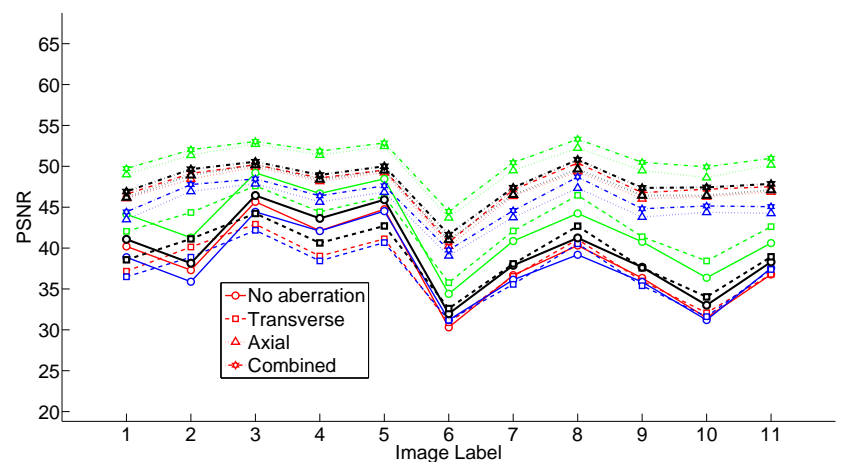

Fig. 6. PSNR computed from straight comparisons between distorted source images and distorted demosaicked images for $\mathrm{R} / \mathrm{G} / \mathrm{B}$ channels and the average with gradient corrected linear interpolation.

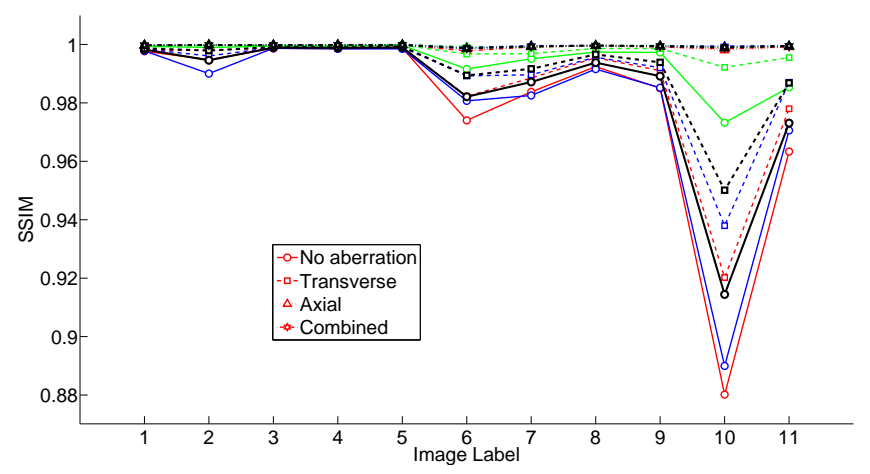

Fig. 7. SSIM indices computed from straight comparisons between distorted source images and distorted demosaicked images for R/G/B channels and the average with bilinear interpolation.

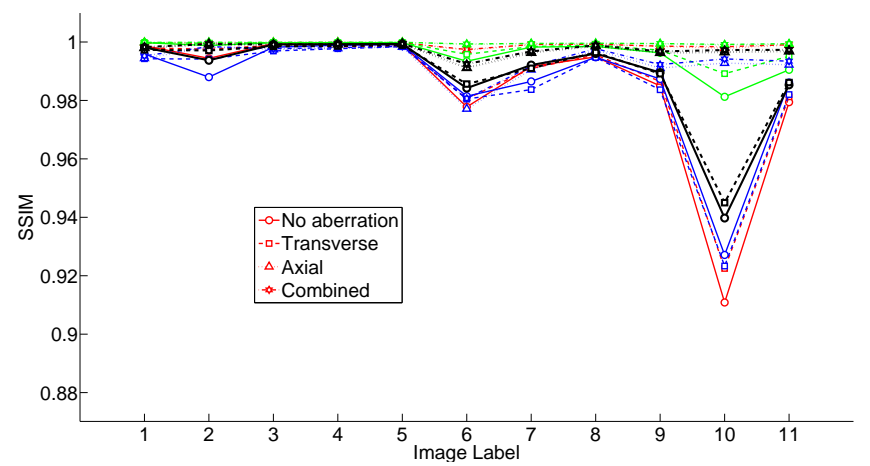

Fig. 8. SSIM indices computed from straight comparisons between distorted source images and distorted demosaicked images for R/G/B channels and the average with gradient corrected linear interpolation.

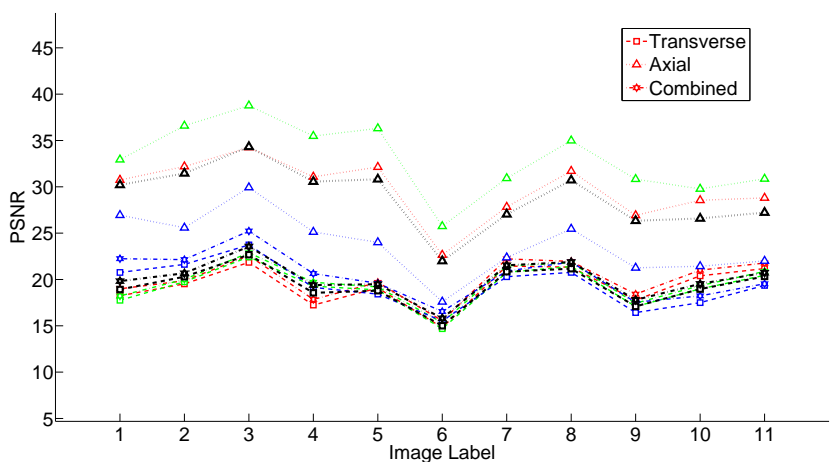

Fig. 9. PSNR computed from cross comparisons between non-distorted source images and distorted demosaicked images for $\mathrm{R} / \mathrm{G} / \mathrm{B}$ channels and the average with gradient corrected linear interpolation.

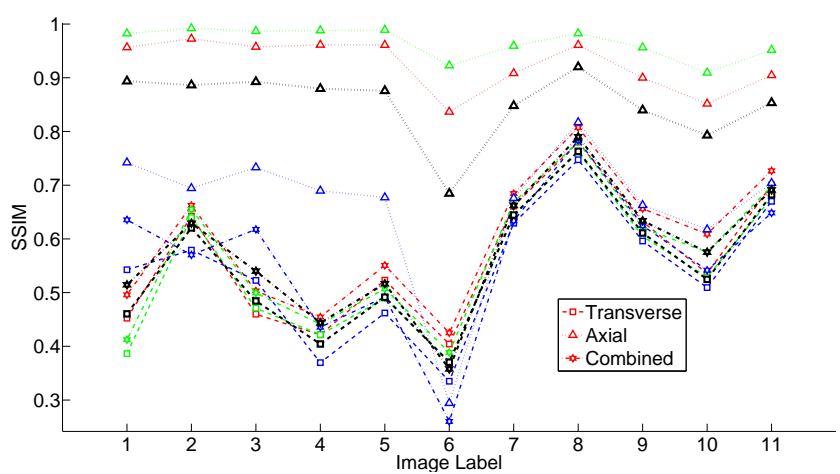

Fig. 10. PSNR computed from cross comparisons between non-distorted source image and distorted demosaicked image for $\mathrm{R} / \mathrm{G} / \mathrm{B}$ channels and the average with gradient corrected linear interpolation.

methods used. This is true for all red, green and blue channels. However, in Fig. 5 the best result was achieved by the blue channel under axial and combined aberrations, whereas in Fig. 6 for the green channel under the same aberrations. Such findings are consistent with Fig. 7-8. This indicates that CA surprisingly benefit demosaicking. In particular, blur benefit both demosaicking methods by higher spatial correlation. In theory, transverse CA in form of mis-registration should reduce correlation, thus decreasing the performance. On the contrary, the figures show opposite results. Perhaps the resampling involved blurs the images to some extent as well. In comparison with intra-channel methods, interchannel interpolation is more sensitive to the content of the green channel. The drop of Image 10 in Fig. 7-8 may be because of the specific characters of the image, i.e., oil painting as the foreground on top of black background. Furthermore, the two distinct models of CA simulation may also affect the results.

Suggested by Fig. 9 and Fig. 10, axial CA results in higher 
performance in terms of cross comparison, indicating that blur influences less the signal fidelity and similarity. In comparison, both of the other two types of CA involve transversal $\mathrm{CA}$ and lead to significantly worse results. Obviously CA lowers the overall image quality by any means.

\section{CONCLUSION}

In this paper we propose a framework to investigate the influence of chromatic aberration (CA) on demosaicking. Experimental results show that $\mathrm{CA}$ benefits demosaicking to some extent, however any type of CA decreases image quality by means of blur and mis-registration.

Certainly the results deserve further study. More demosaicking algorithms and sample images would be helpful. And experiments in subjective assessment of image quality are expected to be conducted, which may reveal better how human observers perceive the issue. Further, the degree of CA may be varied to obtain more data, and ray tracing analysis of real lenses is expected to make the simulation more realistic.

\section{REFERENCES}

[1] B. E. Bayer, “Color imaging array," July 20 1976, US Patent 3,971,065.

[2] H.-C. Lee, Introduction to Color Imaging Science, Cambridge University Press, 2005.

[3] "ISETBIO toolbox," https://github.com/ isetbio/isetbio, Retrieved 20 Jun 2014.

[4] L. N. Thibos, A. Bradley, and X. Zhang, "Effect of ocular chromatic aberration on monocular visual performance," Optometry \& Vision Science, vol. 68, no. 8, pp. 599-607, 1991.

[5] L. N. Thibos, M. Ye, X. Zhang, and A. Bradley, "The chromatic eye: a new reduced-eye model of ocular chromatic aberration in humans," Appl. Opt., vol. 31, no. 19, pp. 3594-3600, Jul 1992.

[6] P. Y. Maeda, “Zernike polynomials and their use in describing the wavefront aberrations of the human eye," http://scien.stanford.edu/pages/ labsite/2003/psych221/projects/03/ pmaeda/, 2003, Retrieved 21 Jun 2014.

[7] J. W. Goodman, Introduction to Fourier Optics, McGraw-Hill physical and quantum electronics series. Roberts \& Company, 2005.

[8] J. E. Farrell, P. B. Catrysse, and B. A. Wandell, "Digital camera simulation," Appl. Opt., vol. 51, no. 4, pp. A80A90, Feb 2012.
[9] H. S. Malvar, L.-W. He, and R. Cutler, "High-quality linear interpolation for demosaicing of bayer-patterned color images," in Proceedings of IEEE International Conference on Acoustics, Speech, and Signal Processing, 2004. (ICASSP '04)., 2004, vol. 3, pp. 485-488.

[10] "Kodak lossless true color image suite," http:// rok.us/graphics/kodak/, PhotoCD PCD0992.

[11] P. Longère, X. Zhang, P. B. Delahunt, and D. H. Brainard, "Perceptual assessment of demosaicing algorithm performance," Proceedings of the IEEE, vol. 90, no. 1, pp. 123-132, Jan 2002.

[12] W. Lu and Y.-P. Tan, "Color filter array demosaicking: new method and performance measures," IEEE Transactions on Image Processing, vol. 12, no. 10, pp. 11941210, Oct. 2003.

[13] Y. Yang, O. Losson, and L. Duvieubourg, “Quality evaluation of color demosaicing according to image resolution," in Third International IEEE Conference on Signal-Image Technologies and Internet-Based System, 2007. SITIS '07., Dec 2007, pp. 689-695.

[14] X. Zhang and B. A. Wandell, "A spatial extension of CIELAB for digital color image reproduction," Journal of the Society for Information Display, vol. 5, no. 3, pp. 61-63, Mar. 1997.

[15] O. Losson, L. Macaire, and Y. Yang, "Comparison of color demosaicing methods," in Advances in Imaging and electron Physics, Peter W. Hawkes, Ed., vol. 162 of Advances in Imaging and Electron Physics, pp. 173265. Elsevier, 2010.

[16] Z. Wang, A. C. Bovik, H. R. Sheikh, and E. P. Simoncelli, "Image quality assessment: from error visibility to structural similarity," IEEE Transactions on Image Processing, vol. 13, no. 4, pp. 600-612, 2004.

[17] D. H. Foster, K. Amano, S. M. C. Nascimento, and M. J. Foster, "Frequency of metamerism in natural scenes," $J$. Opt. Soc. Am. A, vol. 23, no. 10, pp. 2359-2372, Oct 2006.

[18] F. Yasuma, T. Mitsunaga, D. Iso, and S. K. Nayar, "Generalized assorted pixel camera: Postcapture control of resolution, dynamic range and spectrum," Tech. Rep., Department of Computer Science, Columbia University CUCS-061-08, Nov 2008, http: / / www1 . cs . columbia.edu/CAVE / databases/multispectral/.

[19] "The ssim index for image quality assessment," http: //www. cns.nyu.edu/lcv/ssim/, Retrieved 10 Sep 2014. 